\title{
Data Resources for the Wyoming Landscape Conservation Initiative (WLCl) Integrated Assessment (IA)
}

\author{
GIS Data Download
}

\section{U.S. Geological Survey Data Series: http://pubs.usgs.gov/ds/700/}

Authors: Timothy J. Assal, Steven L. Garman, Zachary H. Bowen, Patrick J. Anderson, Daniel Manier, and Robert R. McDougal

\section{U.S. Geological Survey ScienceBase Data Catalog (Data Repository):}

https://www.sciencebase.gov/catalog/folder/4f4e4770e4b07f02db47e1a9

Data associated with the Resource Index:

https://www.sciencebase.gov/catalog/folder/4f4e4770e4b07f02db47e1aa

Data associated with the Condition assessment:

https://www.sciencebase.gov/catalog/folder/4f4e4770e4b07f02db47e1b5

Data associated with the Multicriteria Index:

https://www.sciencebase.gov/catalog/folder/4f4e4771e4b07f02db47e1d6

Data associated with the Change Agents assessment:

https://www.sciencebase.gov/catalog/folder/4f4e4770e4b07f02db47e1c6

Data associated with the Future Change assessment:

https://www.sciencebase.gov/catalog/folder/4f4e4770e4b07f02db47e1cd

Data associated with the WLCl Subwatershed assessment:

https://www.sciencebase.gov/catalog/folder/4f4e4771e4b07f02db47e1d7 